Supporting Information

\title{
Illumination-Induced Phase Segregation and Suppressed Solubility Limit in Br-Rich Mixed-Halide Inorganic Perovskites
}

Yutao Wang, ${ }^{\dagger}$ Xinwei Guan, ${ }^{\dagger}$ Weijian Chen,,$\downarrow$ Jack Yang, ${ }^{\dagger+*}$ Long Hu,${ }^{\dagger}$ Jiong Yang, ${ }^{\perp}$ Sean Li, ${ }^{\dagger}$ Kourosh Kalantar-Zadeh, ${ }^{\perp}$ Xiaoming Wen, ${ }^{\ddagger}$ and Tom $\mathrm{Wu}^{\dagger *}$

${ }^{\dagger}$ School of Materials Science and Engineering and Advanced Materials and Manufacturing Futures Institute, University of New South Wales (UNSW), Sydney, NSW 2052, Australia.

tCentre for Translational Atomaterials, Swinburne University of Technology, Hawthorn, Victoria 3122, Australia

${ }^{\perp}$ School of Chemical Engineering, University of New South Wales (UNSW), Sydney, NSW 2052, Australia.

${ }^{+}$Australian Nuclear Science and Technology Organization, Lucas Heights, NSW, 2234, Australia

E-mail: tom.wu@unsw.edu.au; jianliang.yang1@unsw.edu.au 
Table S1. PL evolution of mixed halide inorganic perovskite thin films and the proposed mechanisms.

\begin{tabular}{|c|c|c|c|c|c|}
\hline $\begin{array}{l}\text { Perovskite } \\
\text { composition }\end{array}$ & PL peak evolution & Reversibility & Mechanism & Year & Ref \\
\hline $\mathrm{CsPbBrI}_{2}$ & stable & / & l & 2016 & {$[1]$} \\
\hline $\mathrm{CsPb}\left(\mathrm{Br}_{0.6} \mathrm{I}_{0.4}\right)_{3}$ & $\begin{array}{l}\text { red shift from } 602 \mathrm{~nm} \\
\text { to } 649 \mathrm{~nm}\end{array}$ & / & / & 2016 & {$[1]$} \\
\hline $\begin{array}{c}\mathrm{CsPbBr}_{2} \mathrm{I} \& \\
\mathrm{CsPbBrI}_{2}\end{array}$ & stable & l & $\begin{array}{l}\text { light-independent } \\
\text { migration barrier }\end{array}$ & 2017 & {$[2]$} \\
\hline $\mathrm{CsPb}\left(\mathrm{Br}_{0.1} \mathrm{I}_{0.9}\right)_{3}$ & $\begin{array}{l}\text { red shift from } 540 \mathrm{~nm} \\
\text { to } 590 \mathrm{~nm}\end{array}$ & / & $\begin{array}{l}\text { electron-phonon } \\
\text { coupling }\end{array}$ & 2017 & {$[3]$} \\
\hline $\mathrm{CsPb}\left(\mathrm{Br}_{0.5} \mathrm{I}_{0.5}\right)_{3}$ & $\begin{array}{l}\text { red shift from } 630 \mathrm{~nm} \\
\text { to } 690 \mathrm{~nm}\end{array}$ & 1 & $\begin{array}{l}\text { bandgap reduction } \\
\text { of I-rich phase }\end{array}$ & 2017 & {$[4]$} \\
\hline $\operatorname{CsPb}\left(\mathrm{Br}_{\mathrm{x}} \mathrm{I}_{1-\mathrm{x}}\right)_{3}$ & shift to $1.87 \mathrm{eV}$ & / & $\begin{array}{l}\text { thermodynamic } \\
\text { preference }\end{array}$ & 2019 & {$[5]$} \\
\hline $\mathrm{CsPb}\left(\mathrm{Br}_{0.9} \mathrm{I}_{0.1}\right)_{3}$ & $\begin{array}{l}\text { blue shift from } 540 \mathrm{~nm} \\
\text { to } 530 \mathrm{~nm}\end{array}$ & irreversible & / & l & $\begin{array}{l}\text { This } \\
\text { work }\end{array}$ \\
\hline $\mathrm{CsPb}\left(\mathrm{Br}_{0.95} \mathrm{I}_{0.05}\right)_{3}$ & $\begin{array}{l}\text { blue shift from } 533 \mathrm{~nm} \\
\text { to } 530 \mathrm{~nm}\end{array}$ & / & l & l & $\begin{array}{l}\text { This } \\
\text { work }\end{array}$ \\
\hline $\mathrm{CsPb}\left(\mathrm{Br}_{0.975} \mathrm{I}_{0.025}\right)_{3}$ & stable & / & 1 & l & $\begin{array}{l}\text { This } \\
\text { work }\end{array}$ \\
\hline $\mathrm{CsPb}\left(\mathrm{Br}_{0.875} \mathrm{I}_{0.125}\right)_{3}$ & rise at $670 \mathrm{~nm}$ & / & l & 1 & $\begin{array}{l}\text { This } \\
\text { work }\end{array}$ \\
\hline $\mathrm{CsPb}\left(\mathrm{Br}_{0.8} \mathrm{I}_{0.2}\right)_{3}$ & $\begin{array}{c}\text { quench at } 557 \mathrm{~nm} \text {, rise } \\
\text { at } 680 \mathrm{~nm}\end{array}$ & reversible & l & l & $\begin{array}{l}\text { This } \\
\text { work }\end{array}$ \\
\hline $\operatorname{CsPb}\left(\mathrm{Br}_{0.67} \mathrm{I}_{0.33}\right)_{3}$ & $\begin{array}{c}\text { quench at } 584 \mathrm{~nm} \text {, rise } \\
\text { at } 673 \mathrm{~nm}\end{array}$ & reversible & I & l & $\begin{array}{l}\text { This } \\
\text { work }\end{array}$ \\
\hline
\end{tabular}


Table S2. Summary of the (100) peak position and the calculated lattice parameter of $\mathrm{CsPb}\left(\mathrm{Br}_{0.9} \mathrm{I}_{0.1}\right)_{3}, \mathrm{CsPb}\left(\mathrm{Br}_{0.8} \mathrm{I}_{0.2}\right)_{3}$, and $\mathrm{CsPb}\left(\mathrm{Br}_{0.67} \mathrm{I}_{0.33}\right)_{3}$ materials according to the XRD patterns.

\begin{tabular}{cccc}
\hline Composition & $2 \theta$ & $\sin \theta$ & $d(\AA)$ \\
\hline $\mathrm{CsPb}\left(\mathrm{Br}_{0.9} \mathrm{I}_{0.1}\right)_{3}$ & 15.23 & 0.1323 & 5.82 \\
$\mathrm{CsPb}\left(\mathrm{Br}_{0.8} \mathrm{I}_{0.2}\right)_{3}$ & 15.00 & 0.1305 & 5.9 \\
$\mathrm{CsPb}\left(\mathrm{Br}_{0.67} \mathrm{I}_{0.33}\right)_{3}$ & 14.94 & 0.13 & 5.92 \\
\hline
\end{tabular}
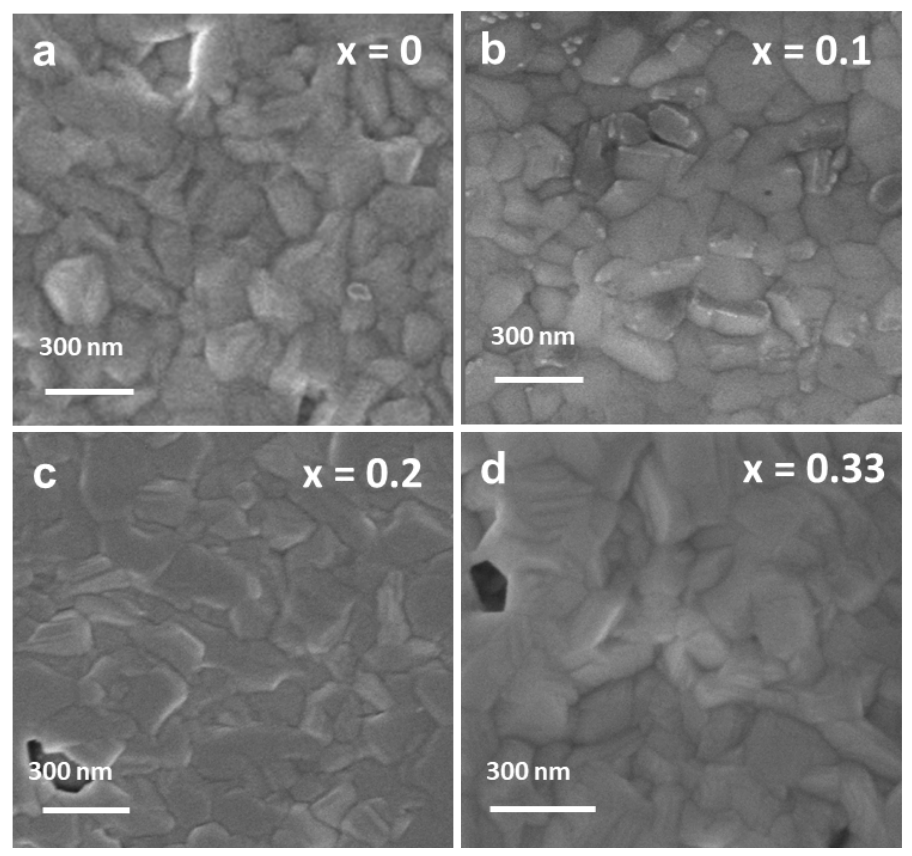

Figure S1. SEM images of films with (a) $\mathrm{x}=0$, (b) $\mathrm{x}=0.1$, (c) $\mathrm{x}=0.2$, (d) $\mathrm{x}=0.33$. 

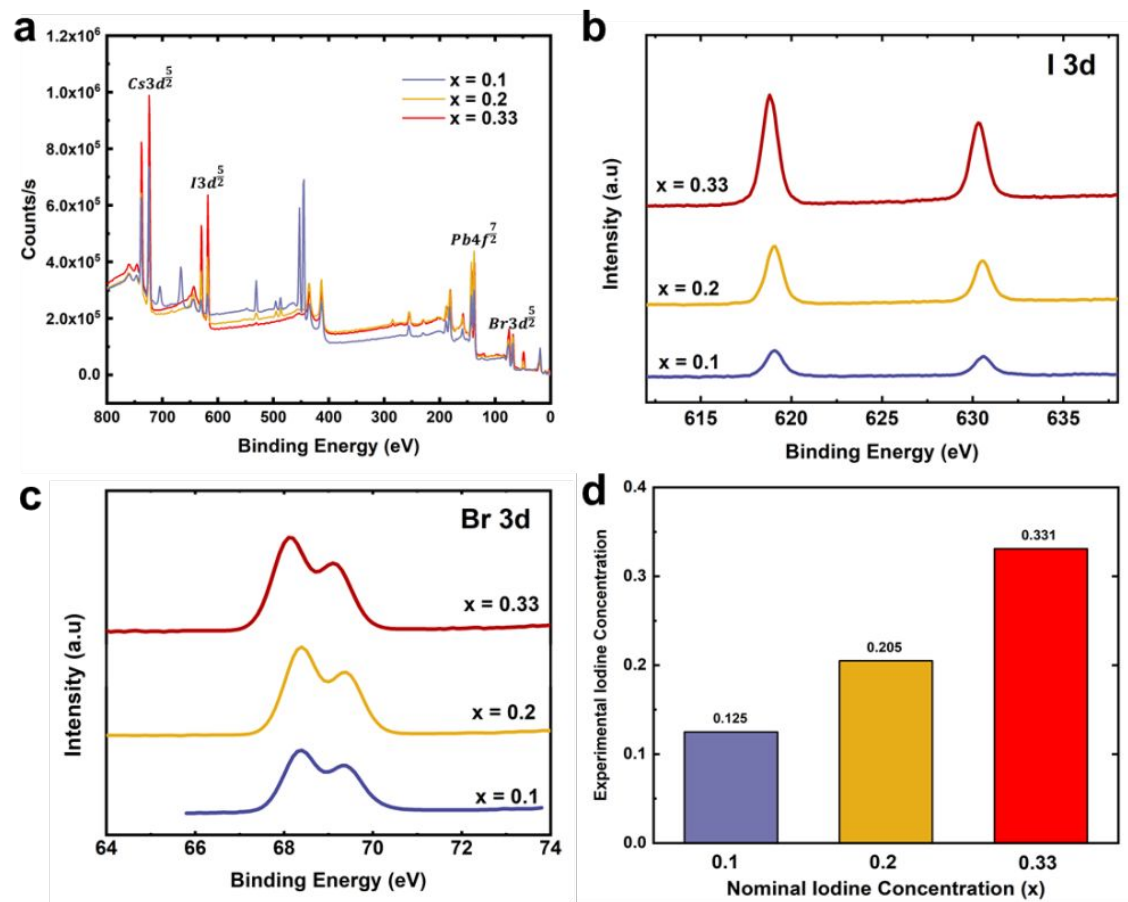

Figure S2. (a) XPS spectra of $\operatorname{CsPb}\left(\mathrm{Br}_{1-\mathrm{x}} \mathrm{I}_{\mathrm{x}}\right)_{3}$ films with $\mathrm{x}=0.1$ (blue line), 0.2 (yellow line) and 0.33 (red line). Characteristic peak of the (b) I 3d and (c) $\mathrm{Br} 3 \mathrm{~d}$ orbital of films with $\mathrm{x}=0.1,0.2$ and 0.33. (d) Calculated iodine concentrations based on the XPS results in different nominal iodine concentrations.

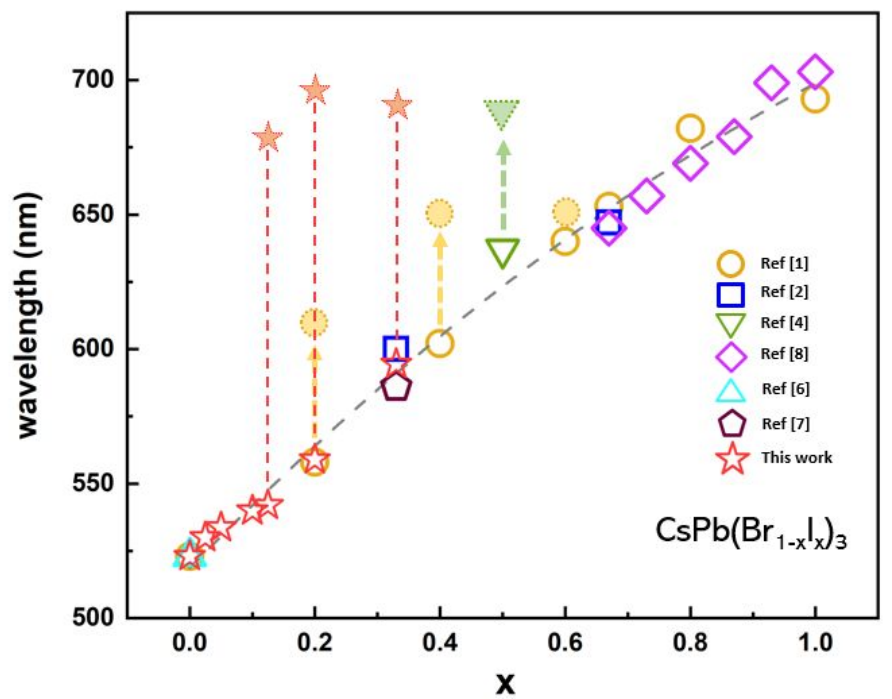

Figure S3. Summarized PL investigations of all-inorganic mixed halide perovskite including this work. ${ }^{[1,2,4,6-8]}$ The shaded pattern represents the PL peak position after illumination. 

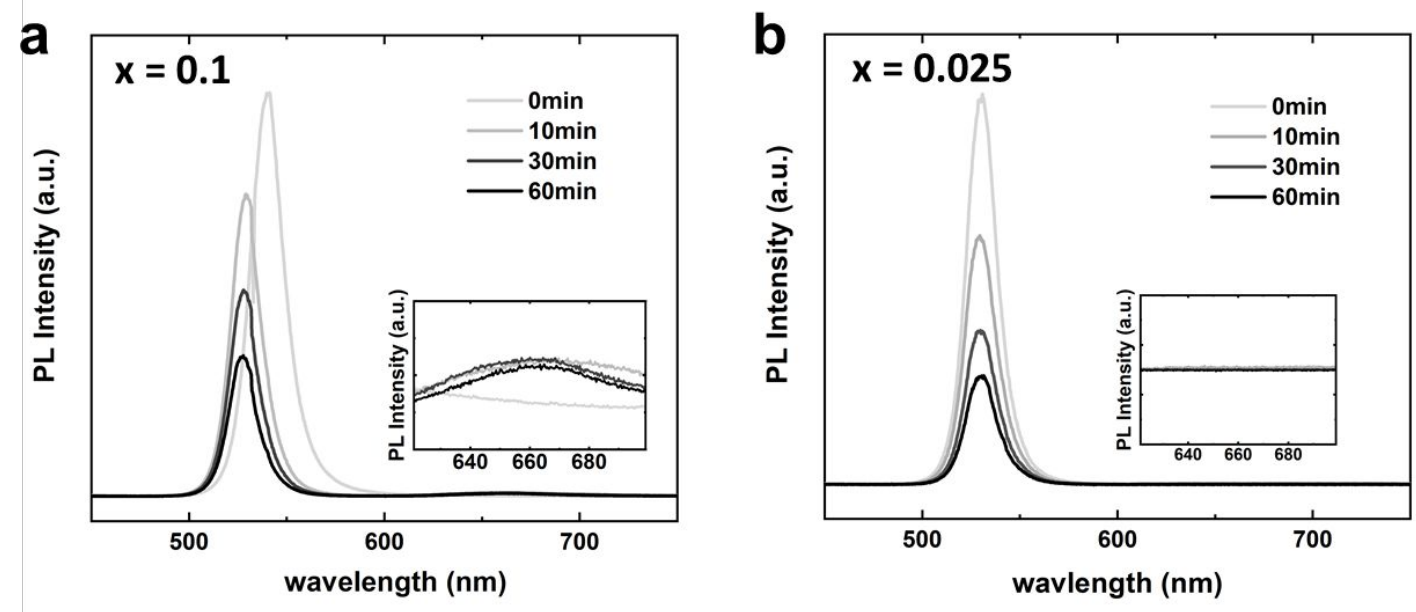

Figure S4. Time-dependent PL spectra of the films with (a) $x=0.1$ and (b) 0.025 measured for one hour. Inset: enlarged scale of the spectra from $620 \mathrm{~nm}$ to $700 \mathrm{~nm}$.
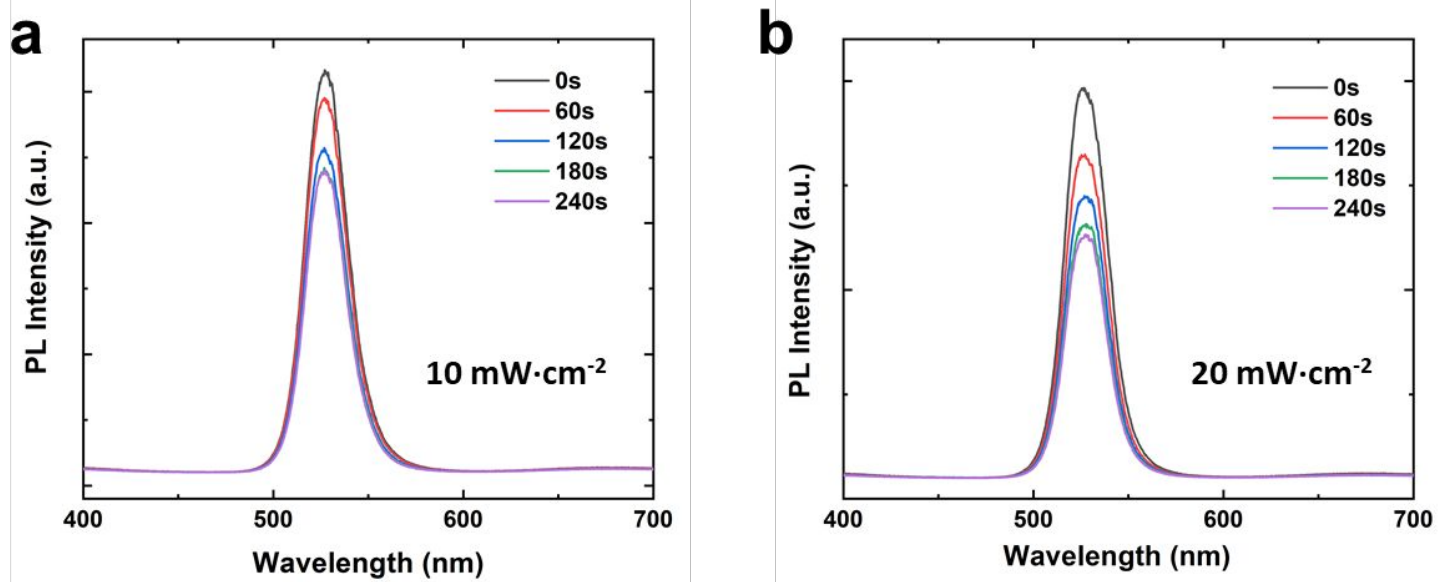

Figure S5. Intensity-dependent PL spectra of the $x=0.025$ film with the excitation intensity of (a) $10 \mathrm{~mW} / \mathrm{cm}^{2}$ and (b) $20 \mathrm{~mW} / \mathrm{cm}^{2}$. The measurement duration is 240 seconds. 

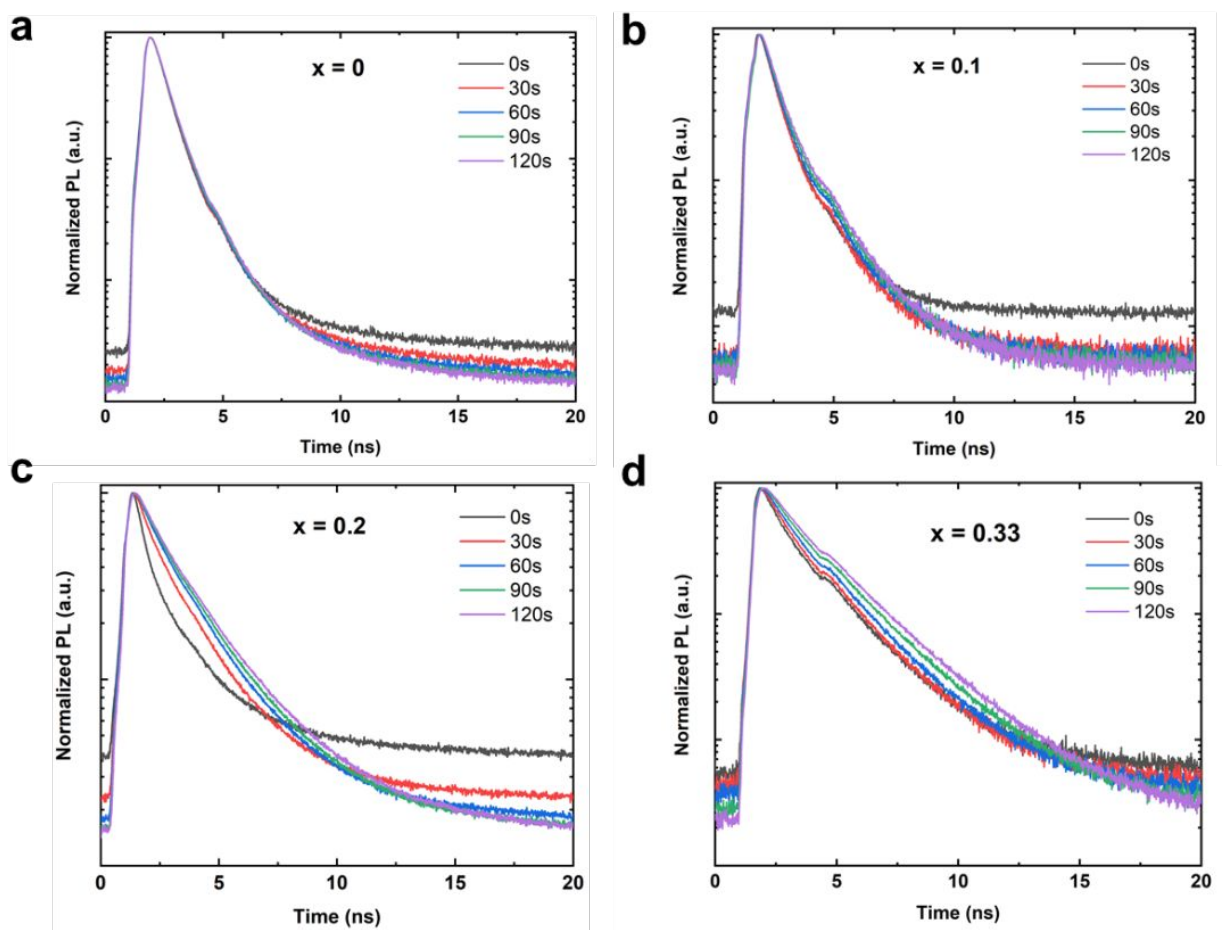

Figure S6. Time resolved PL traces of the samples with (a) $x=0$, (b) $x=0.1$, (c) $x=0.2$ and (d) $x=0.33$ under laser illumination for a consecutive period of $120 \mathrm{~s}$, with each trace cumulated for an interval of $30 \mathrm{~s}$.

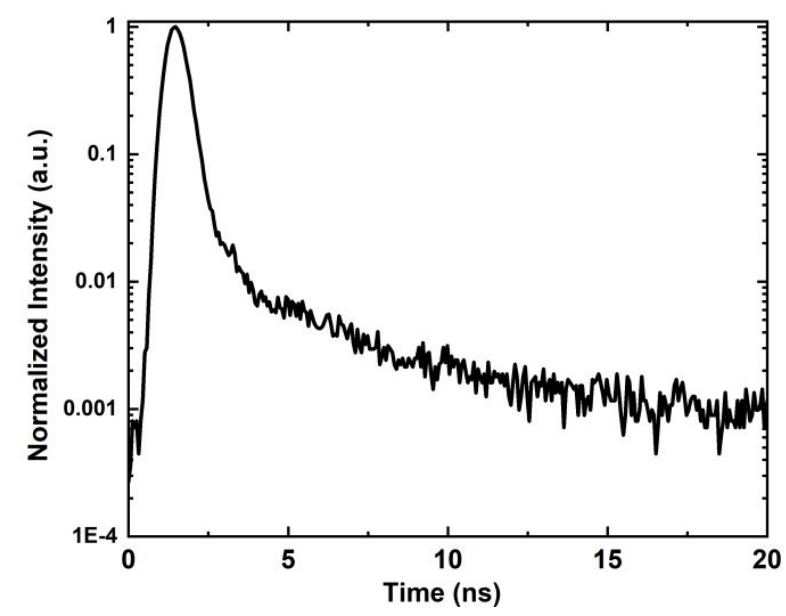

Figure S7. System response function of the TRPL instrument. 


\section{References}

1. Beal, R. E.; Slotcavage, D. J.; Leijtens, T.; Bowring, A. R.; Belisle, R. A.; Nguyen, W. H.; Burkhard, G. F.; Hoke, E. T.; McGehee, M. D., Cesium Lead Halide Perovskites with Improved Stability for Tandem Solar Cells. J. Phys. Chem. Lett 2016, 7, 746-751.

2. Zhou, W.; Zhao, Y.; Zhou, X.; Fu, R.; Li, Q.; Zhao, Y.; Liu, K.; Yu, D.; Zhao, Q., Light-Independent Ionic Transport in Inorganic Perovskite and Ultrastable Cs-Based Perovskite Solar Cells. J. Phys. Chem. Lett 2017, 8, 4122-4128.

3. Bischak, C. G.; Hetherington, C. L.; Wu, H.; Aloni, S.; Ogletree, D. F.; Limmer, D. T.; Ginsberg, N. S., Origin of Reversible Photoinduced Phase Separation in Hybrid Perovskites. Nano Lett. 2017, 17, 1028-1033.

4. $\quad$ Draguta, S.; Sharia, O.; Yoon, S. J.; Brennan, M. C.; Morozov, Y. V.; Manser, J. S.; Kamat, P. V.; Schneider, W. F.; Kuno, M., Rationalizing The Light-Induced Phase Separation of Mixed Halide Organic-Inorganic Perovskites. Nature communications 2017, $8,200$.

5. Wang, X.; Ling, Y.; Lian, X.; Xin, Y.; Dhungana, K. B.; Perez-Orive, F.; Knox,

J.; Chen, Z.; Zhou, Y.; Beery, D.; Hanson, K.; Shi, J.; Lin, S.; Gao, H., Suppressed Phase Separation of Mixed-Halide Perovskites Confined in Endotaxial Matrices. Nature Communications 2019, 10, 695.

6. $\quad$ Li, C.; Zang, Z.; Han, C.; Hu, Z.; Tang, X.; Du, J.; Leng, Y.; Sun, K., Highly Compact $\mathrm{CsPbBr}_{3}$ Perovskite Thin Films Decorated By ZnO Nanoparticles for Enhanced Random Lasing. Nano Energy 2017, 40, 195-202. 
7. Lin, J.; Lai, M.; Dou, L.; Kley, C. S.; Chen, H.; Peng, F.; Sun, J.; Lu, D.; Hawks, S. A.; Xie, C., Thermochromic Halide Perovskite Solar Cells. Nature materials $2018,17,261$.

8. Sutton, R. J.; Eperon, G. E.; Miranda, L.; Parrott, E. S.; Kamino, B. A.; Patel, J. B.; Hörantner, M. T.; Johnston, M. B.; Haghighirad, A. A.; Moore, D. T., BandgapTunable Cesium Lead Halide Perovskites with High Thermal Stability For Efficient Solar Cells. Adv. Energy Mater. 2016, 6, 1502458. 